Check for updates

Cite this: Chem. Sci., 2018, 9, 8402

๑ All publication charges for this article have been paid for by the Royal Society of Chemistry

Received 31st July 2018

Accepted 9th September 2018

DOI: $10.1039 / \mathrm{c} 8 \mathrm{sc} 03393 \mathrm{e}$

rsc.li/chemical-science

\section{Ultrathin two-dimensional covalent organic framework nanoprobe for interference-resistant two-photon fluorescence bioimaging $\dagger$}

\author{
Peng Wang, Fang Zhou, Cheng Zhang, Sheng-Yan Yin, Lili Teng, Lanlan Chen, \\ Xiao-Xiao Hu, Hong-Wen Liu, (D) Xia Yin* and Xiao-Bing Zhang (D)*
}

\begin{abstract}
The complex environment of living organisms significantly challenges the selectivity of classic smallmolecule fluorescent probes for bioimaging. Due to their predesigned topological structure and engineered internal pore surface, covalent organic frameworks (COFs) have the ability to filter out coexisting interference components and help to achieve accurate biosensing. Herein, we propose an effective interference-resistant strategy by creating a COF-based hybrid probe that combines the respective advantages of COFs and small-molecule probes. As a proof of concept, a two-photon fluorescent COF nanoprobe, namely TpASH-NPHS, is developed for targeting hydrogen sulfide $\left(\mathrm{H}_{2} \mathrm{~S}\right)$ as a model analyte. TpASH-NPHS exhibits limited cytotoxicity, excellent photostability and long-term bioimaging capability. More importantly, compared with the small-molecule probe, TpASH-NPHS achieves accurate detection without the interference from intracellular enzymes. This allows us to monitor the levels of endogenous $\mathrm{H}_{2} \mathrm{~S}$ in a mouse model of cirrhosis.
\end{abstract}

\section{Introduction}

Fluorescence imaging is a powerful technique to monitor physiological and pathological processes and to study the functions of biomolecules in their native environment by virtue of its high sensitivity, non-destructive and fast response, and capacity for real-time analysis..$^{1-3}$ In the past few decades, smallmolecule fluorescent probes have been widely applied for the detection and imaging of various biological targets. The fluorescent properties of probes could be tuned via structural adaptation. ${ }^{4,5}$ This allowed for the design of probes showing significant changes in their spectroscopic properties upon interaction with their target analytes. However, the effective biosensing performance of a given small-molecule fluorescent probe can turn out to be quite susceptible to the complex biological environment. In particular, intricate intracellular enzymes may challenge the selectivity and sensitivity of the designed probe in question. For example, cytochrome P450 enzymes (CYP450) can lead to the reduction of azide groups in live cells under hypoxic conditions, which impacts the availability of azide-containing probes for the detection of

Molecular Science and Biomedicine Laboratory, State Key Laboratory of Chemo/Biosensing and Chemometrics, College of Chemistry and Chemical Engineering, College of Life Sciences, Collaborative Innovation Center for Chemistry and Molecular Medicine, Hunan University, Changsha, Hunan 410082, China. E-mail:xbzhang@hnu.edu.cn

$\dagger$ Electronic supplementary information (ESI) available. See DOI: $10.1039 / \mathrm{c} 8 \mathrm{sc} 03393 \mathrm{e}$ intracellular hydrogen sulfide $\left(\mathrm{H}_{2} \mathrm{~S}\right) .{ }^{6}$ Moreover, some limitations of classic small-molecule fluorescent probes, such as unsatisfactory signal stability and/or heterogeneous intracellular distribution, may cause a biosensing application to fail, including any attempt for long-term bioimaging. ${ }^{7,8}$ Therefore, any strategy with the potential to overcome these limitations and realize interference-resistant and long-term imaging is worthy of exploration.

We come to focus on covalent organic frameworks (COFs) as such a platform to solve the abovementioned problems. COFs are an emerging class of organic, porous and crystalline materials that exemplify the power of reticular chemistry and expand the range of materials for real-world applications. ${ }^{9-15}$ Due to their predesigned topological structure, high and regular porosity and large surface area, COFs could regulate the enzyme activity by pore-environment control. ${ }^{16}$ We speculate that COFs might also have the potential to circumvent the problems associated with the interference by enzymes as encountered by small-molecule fluorescent probes, because their nanoscale pores can filter biomacromolecules, including enzymes, rather than small biomolecular analytes. Furthermore, COFs have the potential to improve the stability of a given classic smallmolecule fluorescent probe, thereby opening the path to longterm fluorescence imaging.

In this work, we envisaged combining the respective advantages of small-molecule probes and COFs by creating a hybrid probe. As a proof of concept, a reported imine-linked COF, TpASH,${ }^{17}$ was selected and applied to the design of a fluorescent probe, termed TpASH-NPHS, that targets hydrogen sulfide $\left(\mathrm{H}_{2} \mathrm{~S}\right)$ 
as a model analyte present in biological systems. In view of the micron-sized nature of COFs, there don't appear to exist any reports on their use for the detection and imaging of biological analytes in live cells. ${ }^{18-22}$ To obtain an ultrathin, twodimensional (2D) COF nanoprobe by classic solvent-assisted liquid sonication, ${ }^{22-24}$ we take advantage of the subdued $\pi-\pi$ stacking by sequential post-synthesis fluorescence modification. As we will show, this nanoprobe exhibited satisfactory cell uptake and allowed for interference-resistant, long-term imaging in live cells. We will further demonstrate that TpASHNPHS is useful for the investigation of the role of endogenous $\mathrm{H}_{2} \mathrm{~S}$ in counteracting liver cirrhosis.

\section{Results and discussion}

\section{Synthesis of the COF "TpASH"}

As the COF component, we selected the reported imine-linked TpASH. First, this type of COF exhibits irreversible enol-keto tautomerism (Scheme $\mathrm{S}^{\dagger}{ }^{\dagger}$ ) and is characterized by its exceptional chemical stability. ${ }^{17}$ Second, the $p$-toluenesulfonic acid (PTSA)-mediated synthesis of TpASH is mild and convenient ( $90{ }^{\circ} \mathrm{C}, 12$ hours, water) compared to traditional solvothermal methods and could produce highly crystalline porous products and overcome the hurdles in their further bioapplications. Third, the phenolic hydroxyl present in TpASH serves well in the post-synthesis conjugation with a fluorescent probe. This postsynthesis modification could weaken the interlayer $\pi-\pi$ stacking and benefit the formation of nanoscale COFs by solventassisted liquid sonication.

TpASH is prepared via Schiff-base condensation (Fig. 1A) from 1,3,5-triformylphloroglucinol (Tp) and the PTSA salt of 4-aminosalicylhydrazide (ASH) (Scheme S1 and S2 $\dagger$ ). The morphology and size of TpASH were first investigated by transmission electron microscopy (TEM) and field emission scanning electron microscopy (SEM). As shown in Fig. 1B and C, both the TEM and SEM images clearly showed the sheet-like morphology of TpASH. The high-resolution TEM (HRTEM) image showed an interlayer distance of $3.2 \AA$ (Fig. S8†). This indicates a good agreement with the interlayer distance calculated from the (001) diffraction. ${ }^{17}$ PXRD patterns of TpASH showed the first peak at $2 \theta=\sim 3.9^{\circ}$, which corresponds to the reflection from the (100) planes (Fig. S9 $\dagger) .{ }^{17}$ The broad peak at $2 \theta=\sim 26.7^{\circ}$ could be indexed to the $\pi-\pi$ stacked planes (001) of TpASH. ${ }^{17}$ Fourier transform infrared (FTIR) spectra of TpASH exhibited characteristic $\mathrm{C}=\mathrm{C}$ and $\mathrm{C}-\mathrm{N}$ stretching frequencies at $1569 \mathrm{~cm}^{-1}$ and $1250-1257 \mathrm{~cm}^{-1}$, respectively (Fig. 1D).

\section{Post-synthesis fluorescent probe conjugation}

In order to demonstrate the utility of a COF-based biosensor, we selected $\mathrm{H}_{2} \mathrm{~S}$ as a model analyte. As one of the vital gasotransmitters in living organisms, $\mathrm{H}_{2} \mathrm{~S}$ plays a crucial role in many biological processes including neurodegenerative diseases, aging, and tumor proliferation..$^{25-30} \mathrm{~A}$ number of studies have focused on $\mathrm{H}_{2} \mathrm{~S}$ detection with fluorescent probes containing an azide group. ${ }^{31-33}$ Based on our previous work, we chose here to use a two-photon fluorescent probe to

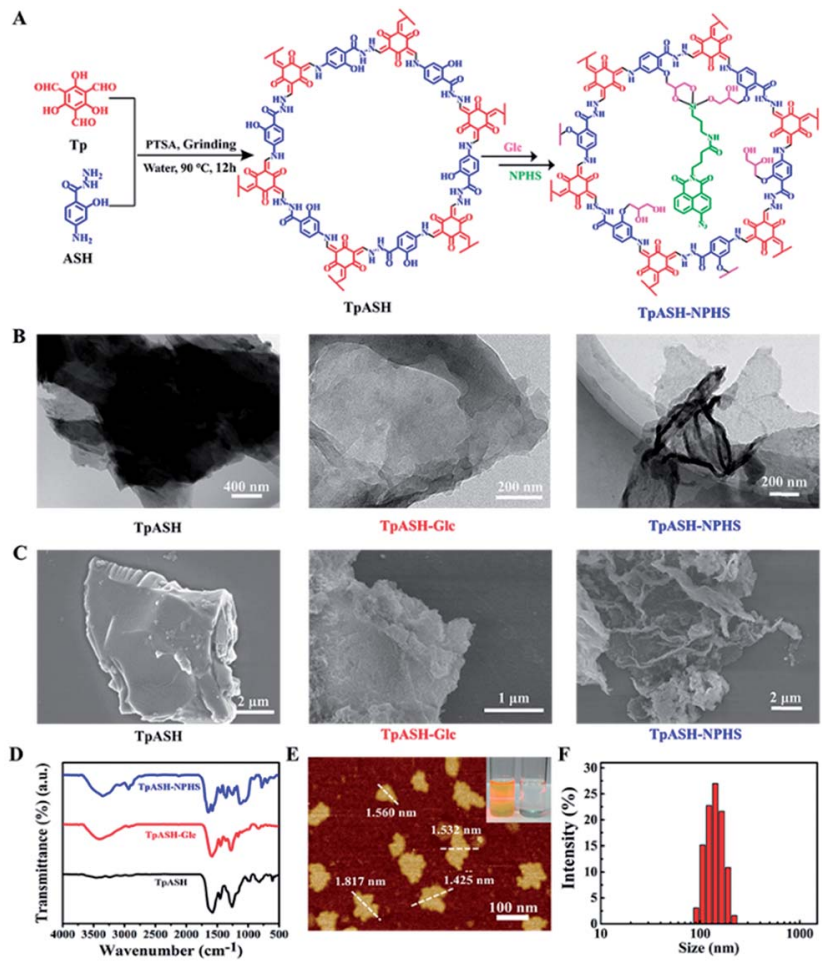

Fig. 1 Construction and characterization of the two-photon fluorescent covalent organic framework nanoprobe TpASH-NPHS. (A) Synthesis of TpASH and its sequential post-synthesis modification. (B) TEM and (C) SEM images of TpASH, TpASH-Glc and TpASH-NPHS. (D) FTIR spectra of TpASH after sequential post-synthesis modification. (E) AFM image of the COF nanoprobe TpASH-NPHS. Inset: the image of the Tyndall effect of the TpASH-NPHS suspension (left: TpASH-NPHS and right: water; source of laser light on the left). (F) The size distribution of the COF nanoprobe TpASH-NPHS by DLS.

functionalize TpASH. ${ }^{31,34-37}$ Two-photon microscopy, which employs near-infrared (NIR) radiation as the excitation source, provides an opportunity for bioimaging with minimal background, reduced photo-damage, better three-dimensional localization and increased penetration depth $(>500 \mu \mathrm{m}))^{3,38,39}$ A 4-amino-1,8-naphthalimide derivative (NPHS) was chosen as the two-photon fluorescent probe by virtue of its large twophoton absorption cross-section, high quantum yield and straightforward synthesis (see Scheme $\mathrm{S} 3 \uparrow$ for an optimized synthetic path). ${ }^{37,40,41}$ We also synthesized a reported $\mathrm{H}_{2} \mathrm{~S}$ fluorescent probe (CNPHS) for comparison (Scheme $\mathrm{S} 4 \dagger$ ).

The phenolic hydroxyl group of TpASH turned out to be a welcome molecular anchor to allow for conjugation with NPHS (Scheme S5 $\dagger$ ). We thus alkylated TpASH by using glycidol (Glc) via epoxide ring opening. The thus established presence of multiple glycols in close vicinity then served to create a strong trivalent link between NPHS (containing a 3-aminopropyltriethoxysilane (APTES) unit) and the COF. In order to confirm the success of this modification, FTIR analysis was performed (Fig. 1D). Compared with TpASH, TpASH-NPHS showed additional peaks corresponding to $\mathrm{C}-\mathrm{H}$ asymmetric and symmetric stretching frequencies at 2920 and $2853 \mathrm{~cm}^{-1}$, respectively, which were attributed to the methylene fragments 
of Glc. In TpASH-NPHS, additional peaks corresponding to $\mathrm{Si}-\mathrm{O}$ stretching bands were also observed at $1095 \mathrm{~cm}^{-1}$ and $1020 \mathrm{~cm}^{-1}$. After fluorescent probe conjugation, the BrunauerEmmett-Teller (BET) surface area of TpASH-NPHS showed a sharp reduction compared to that of TPASH (Fig. S10 $\dagger$ ). The PXRD pattern suggested that TPASH-NPHS maintained the structural integrity of COFs (Fig. S9†). Further analysis by TEM and SEM furnished a direct morphological comparison. As shown in the TEM and SEM images in Fig. 1B and C, TPASHNPHS showed a rippled sheet-like morphology with lateral dimensions in the micron range. This difference between TPASH and TpASH-NPHS is in accord with the notion of postsynthesis weakening of interlayer $\pi-\pi$ stacking. ${ }^{13} \mathrm{C}$ crosspolarization magic-angle spinning (CP/MAS) solid state NMR spectra supported the conjugation of NPHS on the TpASH (Fig. S12 $\dagger$ ). ${ }^{17}$ Energy dispersive X-ray (EDX) analysis associated with TEM demonstrated the successful conjugation by proving the presence of silicon in TPASH-NPHS (Fig. S13 $\dagger$ ). The zeta potential value changed after sequential post-synthesis modification of the COF (Fig. S14 $\dagger$ ). The characteristic peak at $416 \mathrm{~nm}$ (two-photon fluorophore naphthalimide) in the UV-Vis spectra is further evidence for the successful conjugation (Fig. S15†).

\section{Preparation of the COF nanoprobe TpASH-NPHS}

For further bioapplication, nanoscale COFs are desired. However, the obtained COFs are usually micron-sized, which is attributed to the strong interlayer $\pi-\pi$ stacking interactions. ${ }^{\mathbf{4 2 , 4 3}}$ Fortunately, 2D nanosheets can be obtained directly from their bulk counterparts via solvent-assisted exfoliation. As a new member of the family of 2D materials, COF nanosheets may be easily prepared using this strategy. ${ }^{22-24}$ Hence, we take advantage of subdued $\pi-\pi$ stacking by sequential post-synthesis modification to acquire nanoscale COFs by classic solventassisted liquid sonication. After exfoliation, the solution of TPASH-NPHS showed a uniform dispersion and a typical "Tyndall effect" (inset in Fig. 1E), confirming its nanoscale colloidal structure. ${ }^{22}$ The atomic force microscopy (AFM) images in Fig. 1E and S16 $†$ clearly showed a change between the bulk COFs and the nanoscale COFs. Our ultrathin 2D COF TPASH-NPHS showed a size of $87.35 \pm 18.64 \mathrm{~nm}$ and a thickness of $1.58 \pm 0.25 \mathrm{~nm}$, while bulk TpASH-NPHS had a micronscale size and thickness. The average hydrodynamic diameter of TpASH-NPHS was $144.55 \pm 45.59 \mathrm{~nm}$ as determined by dynamic light scattering (DLS) (Fig. 1F). The FTIR spectrum of nanoscale TPASH-NPHS remains the same as that of the bulk material (Fig. S17 $\dagger$ ), further confirming the same structures for the bulk and the nanoscale TpASH-NPHS.

\section{Fluorescence sensing of the nanoprobe TPASH-NPHS}

In order to explore the biosensing performance of the COFbased fluorescent probe, we report here the detection of the naturally occurring analyte $\mathrm{H}_{2} \mathrm{~S}$ by our nanoprobe TPASH-NPHS described above. Its azide units suffer from reduction to amino groups in the presence of $\mathrm{H}_{2} \mathrm{~S}$, which enhances its fluorescence emission (Scheme $\mathrm{S} 7 \dagger$ ). We thus recorded the fluorescence spectral changes in TpASH-NPHS upon the gradual addition of $\mathrm{H}_{2} \mathrm{~S}$. Sodium hydrosulfide ( $\mathrm{NaHS}$ ) was used as the $\mathrm{H}_{2} \mathrm{~S}$ source in all experiments. As shown in Fig. 2B and S18, $\uparrow$ the addition of NaHS triggered a significant increase in fluorescence intensity at $535 \mathrm{~nm}$. This response was linearly proportional to NaHS in the concentration range of $0-20 \mu \mathrm{M}$ (Fig. 2C). The detection limit $(\mathrm{S} / \mathrm{N}=3)$ for NaHS was calculated to be $0.11 \mu \mathrm{M}$, a competitive value compared to previous reports of intracellular $\mathrm{H}_{2} \mathrm{~S}$ detection. ${ }^{31}$ The signal reached a plateau after about $40 \mathrm{~min}$ (Fig. S19†). The loading concentration of NPHS in

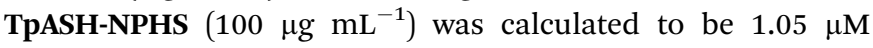
(Fig. S20†).

The selectivity of the COF nanoprobe for $\mathrm{H}_{2} \mathrm{~S}$ was first investigated in vitro. As can be seen in Fig. $2 \mathrm{D}$ and $\mathrm{S} 23, \uparrow$ the fluorescence of TPASH-NPHS and CNPHS was insensitive to the presence of common anions $\left(\mathrm{Cl}^{-}, \mathrm{Br}^{-}, \mathrm{I}^{-}, \mathrm{HCO}_{3}{ }^{-}, \mathrm{HPO}_{4}{ }^{2-}\right.$, $\mathrm{OAc}^{-}, \mathrm{NO}_{2}{ }^{-}, \mathrm{SO}_{4}{ }^{2-}, \mathrm{SO}_{3}{ }^{2-}, \mathrm{HSO}_{3}{ }^{-}, \mathrm{S}_{2} \mathrm{O}_{3}{ }^{2-}$, and $\mathrm{S}_{2} \mathrm{O}_{5}{ }^{2-}$ ), biothiols (glutathione (GSH), cysteine (Cys), and homocysteine (Hcy)) and reactive oxygen species $\left(\mathrm{ClO}^{-}\right.$and $\left.\mathrm{H}_{2} \mathrm{O}_{2}\right)$ under normoxic $\left(21 \% \mathrm{O}_{2}\right)$ and hypoxic $\left(<0.1 \% \mathrm{O}_{2}\right)$ conditions, a result that is in agreement with reported conclusions. ${ }^{31,33}$

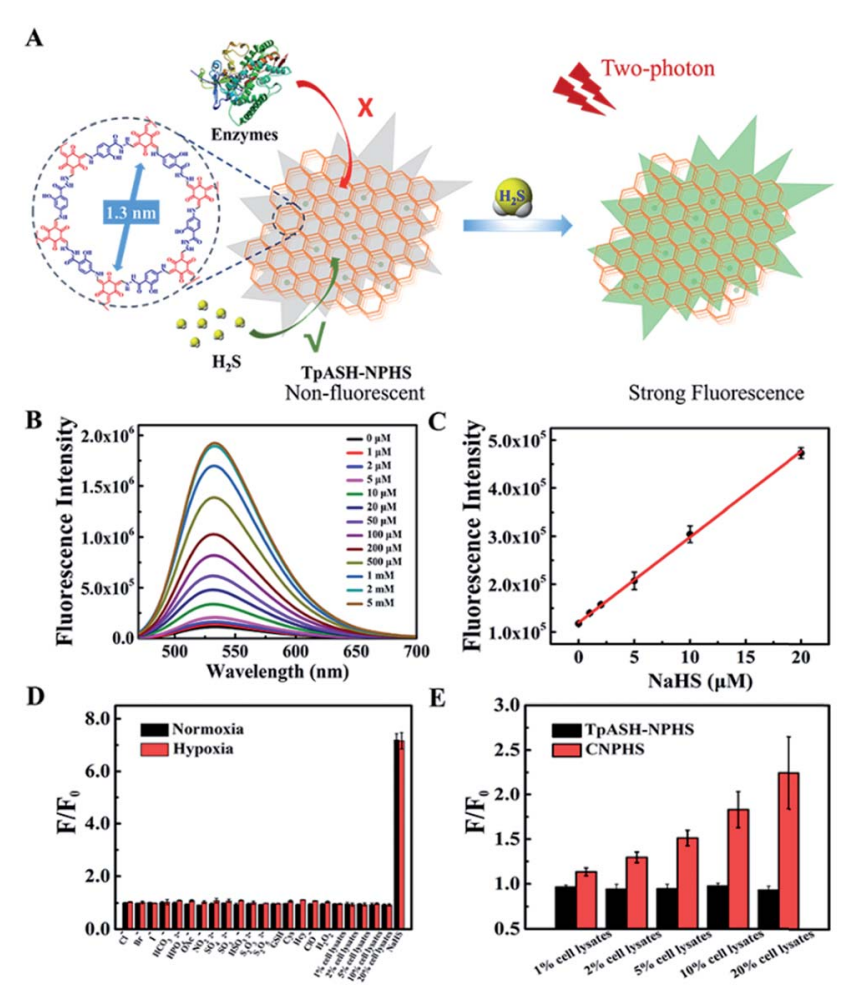

Fig. 2 Interference-resistant fluorescence sensing of the COF nanoprobe TpASH-NPHS. (A) Constitution and mode of action of the COF-based nanoprobe TpASH-NPHS. (B) The fluorescence emission spectra of TpASH-NPHS in the presence of different concentrations of $\mathrm{NaHS}$. (C) Linear response at low NaHS concentrations, $R^{2}=0.998$. The standard curve of the TpASH-NPHS response to NaHS is $y=$ $119908.37448+17831.54373 x . \lambda_{\mathrm{ex}}=440 \mathrm{~nm}$. (D) Fluorescence response of TpASH-NPHS to various substances under normoxic (21\% $\left.\mathrm{O}_{2}\right)$ and hypoxic $\left(<0.1 \% \mathrm{O}_{2}\right)$ conditions. (E) The comparison of fluorescence response between TpASH-NPHS and CNPHS to different concentrations of HepG2 cell lysates under hypoxia. 
However, a recent study reported that intracellular enzymes are able to reduce the azide group under hypoxic conditions. ${ }^{6}$ We suggest here that COFs (pore size of TPASH $=1.3 \mathrm{~nm}$ ) can act as the host material for such a probe, thus denying the access of macromolecular enzymes (usually size $>3 \mathrm{~nm}$ ) to sequestered responsive probes within the COF (Fig. 2A). ${ }^{\mathbf{1 6}, 17}$ To check the interference-resistant ability of our nanoprobe, we investigated the detection performance of TPASH-NPHS in the lysates from HepG2 cells under hypoxic and normoxic conditions. For comparison, experiments with the small-molecule probe (CNPHS) were also conducted. As shown in Fig. 2D, E and S23, $\uparrow$ TpASH-NPHS displayed no obvious fluorescence enhancement in either case, while CNPHS showed strong fluorescence enhancement only for the hypoxic case. This supports the notion that intracellular enzymes are able to cause a false positive signal for $\mathrm{H}_{2} \mathrm{~S} .^{6}$ In addition, both TpASH-NPHS and CNPHS maintained the capacity to respond to the presence of $\mathrm{H}_{2} \mathrm{~S}$ under hypoxic conditions. Thus, we conclude that our COF nanoprobe is able to solve the problem of biomacromolecular interference to which most classic smallmolecule fluorescent probes are susceptible.

\section{Stability and cytotoxicity of TpASH-NPHS}

Due to the irreversible enol-keto tautomerism found in the structure of TpASH (Scheme S6 $\dagger$ ), this type of COF promises elevated chemical stability, which is necessary for bioapplications. DLS profiles taken from PBS $(\mathrm{pH}=7.4)$ and DMEM (cell culture medium) samples support this notion. No significant change in size was found after $24 \mathrm{~h}$ (Fig. S24†), thus demonstrating that the structure of TpASH-NHS remains unaltered in a physiological environment. Next, we investigated the photostability of the nanoscale COFs by monitoring the fluorescence change (Fig. S25 $\dagger$ ). The results showed that the prepared nanoprobe TpASH-NPHS had good photostability in PBS or DMEM for long periods of time. When compared to CNPHS (Fig. S26 $\dagger$ ), the intensity of TPASH-NPHS remains virtually untouched over $3600 \mathrm{~s}$, while that of CNPHS has decreased by 26 percent in just a short period of real time laser scanning. We interpret this to be the fruit of the sheltering effect exercised by the framework architecture of COFs. Identical conclusions were obtained when TpASH-NPHS was tested for fluorescence variations with changing $\mathrm{pH}$ (from 3.0 to 10.0, see Fig. $\mathrm{S} 27 \dagger$ ). Importantly, we also determined the cytotoxicity of TPASH-NPHS by an MTS assay using human cervical carcinoma cells (HeLa) and human liver cancer cells (HepG2). As shown in Fig. S28, $\uparrow$ both the HeLa and HepG2 cells maintained high viability (>90\%) after incubation with TPASH-NPHS for $24 \mathrm{~h}$, even at high concentrations $\left(200 \mu \mathrm{g} \mathrm{mL} \mathrm{m}^{-1}\right)$, clearly indicating the limited cytotoxicity of this COF nanoprobe and suggesting its suitability for intracellular biosensing.

\section{Two-photon fluorescence imaging of $\mathrm{H}_{2} \mathrm{~S}$ in live cells}

The favorable properties of our COF-based probe technology as elucidated above encouraged us to explore its performance during two-photon fluorescence imaging of $\mathrm{H}_{2} \mathrm{~S}$ in live cells. As shown in Fig. 3A, incubation of HepG2 or HeLa cells with

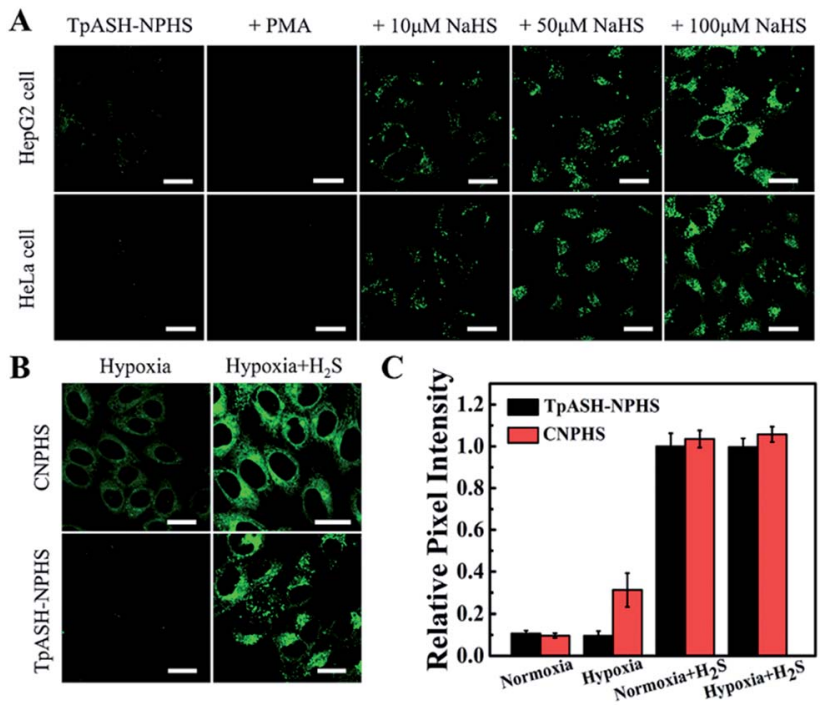

Fig. 3 Interference-resistant imaging of the COF nanoprobe TpASHNPHS in live cells. (A) Two-photon confocal fluorescence imaging of TpASH-NPHS in HepG2 and HeLa cells. Scale bar: $20 \mu \mathrm{m}$. (B) Confocal fluorescence imaging of CNPHS or TpASH-NPHS in HepG2 cells under hypoxic conditions without or with the addition of exogenous $\mathrm{H}_{2} \mathrm{~S}$. Scale bar: $20 \mu \mathrm{m}$. (C) The relative pixel fluorescence intensity of HepG2 cell images. The pixel intensity from TpASH-NPHS incubated with $\mathrm{H}_{2} \mathrm{~S}$ in HepG2 cells under normoxic conditions is defined as 1.0.

TPASH-NPHS afforded a weak green fluorescence signal with two-photon excitation at $820 \mathrm{~nm}$, thereby demonstrating sufficient cell permeability and the promising prospect of endogenous $\mathrm{H}_{2} \mathrm{~S}$ sensing with low background fluorescence in cells. To confirm this, HepG2 and HeLa cells were pretreated with PMA for $30 \mathrm{~min}$, which favors a decrease of endogenous $\mathrm{H}_{2} \mathrm{~S}$ by inducing phagocytosis-associated ROS generation, and then coincubated with the probe before fluorescence imaging. As shown in Fig. 3A, almost no fluorescence was observed. Conversely, the presence of TPASH-NPHS generates a gradually enhanced fluorescence when the cells are co-incubated with NaHS. The mechanism of internalization of TpASH-NPHS was investigated with HepG2 cells. The confocal microscopy images of treatments at $4{ }^{\circ} \mathrm{C}$ (to inhibit energy-dependent endocytosis), or with genistein (a caveolae-dependent endocytosis inhibitor), with chlorpromazine hydrochloride (CPZ, a clathrin-dependent endocytosis inhibitor), and with methyl- $\beta$-cyclodextrin (MBC, a lipid raft-mediated endocytosis inhibitor) are shown in Fig. S29. $\dagger$ The images demonstrate that internalization of TPASH-NPHS is significantly slowed down after the treatment at low temperature or with $\mathrm{CPZ}$, suggesting that the uptake of TpASH-NPHS is mainly through clathrin-dependent endocytosis. We also used the bulk COF TpASH-NPHS for imaging. Poor internalization was evident (Fig. S30†).

Next on our agenda was to determine whether components of the complex intracellular environment could interfere in the sensing and imaging of $\mathrm{H}_{2} \mathrm{~S}$. As shown in Fig. 3B, CNPHS generates dramatically enhanced fluorescence under hypoxic conditions, while TpASH-NPHS does not, which is consistent with the fluorescence response in HepG2 cell lysates under similar conditions. When exogenous $\mathrm{H}_{2} \mathrm{~S}$ is added, both 
TPASH-NPHS and CNPHS show strong fluorescence under normoxic and hypoxic conditions (Fig. 3C and S31†). This proves that the use of TpASH-NPHS over small-molecule responsive probes can prevent enzymes from interfering with the biosensing and bioimaging of $\mathrm{H}_{2} \mathrm{~S}$. To illustrate the difference in the signal stability of fluorescent COF nanoprobes and small-molecule fluorescent probes, we conducted real-time imaging of HepG2 cells in the presence of TPASH-NPHS and CNPHS. Indeed, TpASH-NPHS exhibited enhanced photostability over CNPHS, suggesting that the COF scaffold not only shelters the fluorophore from enzymes but also from photodegradation (Fig. S32†). We now turned to investigating the long-term cellular tracking capability of TpASH-NPHS and CNPHS after different incubation periods. As shown in Fig. S33, $\uparrow$ CNPHS tended to translocate out of cells within one hour, thereby limiting its application to short-term imaging experiments. On the other hand, TpASH-NPHS showed strong intracellular fluorescence at every time point for over six hours.

\section{Two-photon fluorescence imaging of $\mathrm{H}_{2} \mathrm{~S}$ in deep tumor tissues}

The two major advantages of two-photon over single-photon fluorescence imaging are the minimization of tissue autofluorescence and enhanced penetration depth. Fig. S34† reports the data obtained for a two-photon imaging experiment of TPASH-NPHS monitoring $\mathrm{H}_{2} \mathrm{~S}$ in HeLa tumor tissues. Signal intensity vs. scan depth was recorded with a two-photon microscope in the $Z$-scan mode. The control samples without COFs exhibited hardly any auto-fluorescence, demonstrating that two-photon excited confocal images indeed avoid the depiction of auto-fluorescence. On the other hand, tumor tissues incubated with TpASH-NPHS showed a weak fluorescence signal, thus demonstrating that two-photon fluorescent COF nanoprobes are capable of imaging endogenous $\mathrm{H}_{2} \mathrm{~S}$. When such tissue is pretreated with $100 \mu \mathrm{M}$ NaHS, a dramatic fluorescence increase is observed, even at a depth of 50-250 $\mu \mathrm{m}$, which confirms our design objective that TpASH-NPHS is capable of $\mathrm{H}_{2} \mathrm{~S}$ imaging at greater penetration depths. Fig. 4
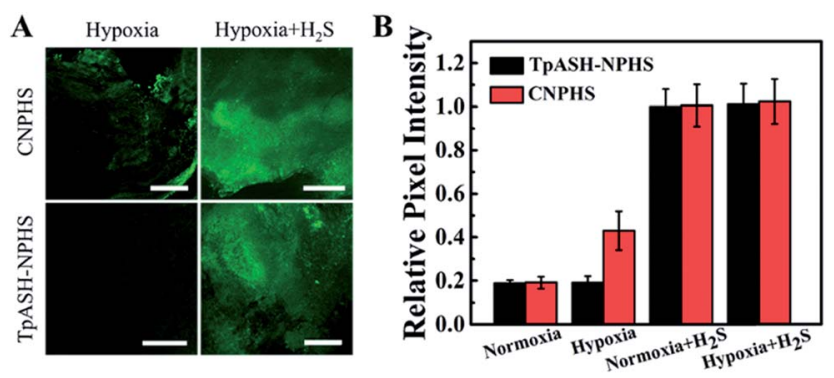

Fig. 4 Interference-resistant two-photon fluorescence imaging of the COF nanoprobe TpASH-NPHS in deep tumor tissues. (A) Twophoton confocal fluorescence images of TpASH-NPHS and CNPHS under hypoxia in HepG2 tumor tissues without or with the addition of exogenous $\mathrm{H}_{2} \mathrm{~S}$. Scale bar: $300 \mu \mathrm{m}$. (B) The relative pixel fluorescence intensity of HepG2 tumor tissues. The pixel signal intensity from TpASH-NPHS incubated with $\mathrm{H}_{2} \mathrm{~S}$ in HepG2 tumor tissues under normoxic conditions is defined as 1.0 . shows the data of imaging experiments on HepG2 tumor tissues. TpASH-NPHS showed no two-photon fluorescence intensity changes under normoxic and hypoxic conditions at a depth of $150 \mu \mathrm{m}$, while CNPHS caused an increase in intensity under hypoxic conditions. The penetration depth of TPASHNPHS-emitted light was also probed in HepG2 tumor tissues (Fig. S35†).

\section{Monitoring of endogenous $\mathrm{H}_{2} \mathrm{~S}$ in a cirrhotic liver model}

As a gasotransmitter with mediating vasodilatory function, endogenous $\mathrm{H}_{2} \mathrm{~S}$ has a significant effect on antagonizing chronic liver injury or cirrhosis. ${ }^{44}$ The levels of endogenous $\mathrm{H}_{2} \mathrm{~S}$ in the liver are a potential physiological index for evaluating the cirrhosis degree. Especially the up-regulated $\mathrm{H}_{2} \mathrm{~S}$ expression at an early stage of cirrhosis benefits the early detection of this liver disease. ${ }^{45}$ However, the liver, as a vital metabolic organ of mammals, possesses the most complex enzyme networks and other components, which impact the biosensing performance of small-molecule fluorescent probes. Besides, a protocol to build a reliable cirrhosis mouse model is needed to subcutaneously inject tetrachloromethane $\left(\mathrm{CCl}_{4}\right)$ to induce hepatotoxicity by CYP2E1 (a member of the CYP450 system) activation. ${ }^{46}$ Therefore, a responsive probe truly specific to $\mathrm{H}_{2} \mathrm{~S}$ sensing would be more than welcome for the bioimaging of the mouse liver with early stage of cirrhosis. The liver tissue slices were harvested at different $\mathrm{CCl}_{4}$-treated periods and stained with TpASH-NPHS (Fig. 5A). As shown in Fig. 5B and S37, $\uparrow$ the twophoton fluorescence intensity steadily increased from the third day (termed 3 day) to the fifteenth day (termed 15 day). This is supported by the observation that the level of endogenous $\mathrm{H}_{2} \mathrm{~S}$ is up-regulated to prevent acute liver injury. ${ }^{\mathbf{4 4 , 4 5}}$ In addition, a liver self-repairing process is indicated by the
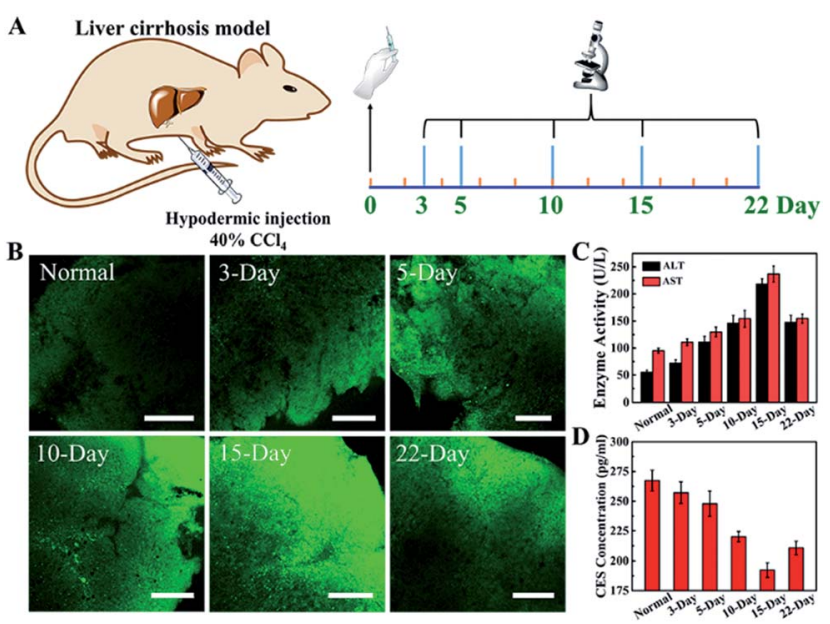

Fig. 5 Monitoring of endogenous $\mathrm{H}_{2} \mathrm{~S}$ in a cirrhotic liver model. (A) The liver cirrhosis mouse model was induced by subcutaneous injection of $40 \% \mathrm{CCl}_{4}$. The liver tissue slices were harvested at different $\mathrm{CCl}_{4}$-treated periods. (B) Two-photon confocal fluorescence images of TpASH-NPHS in liver tissues of the cirrhotic mouse model at different $\mathrm{CCl}_{4}$-treated periods. Scale bar: $300 \mu \mathrm{m}$. (C) The enzyme activity of ALT and AST in mice serum. (D) Quantification of CSE levels in normal and liver cirrhosis models by ELISA. 
increased expression levels of alanine transaminase (ALT) and aspartate transaminase (AST) in hepatocytes (Fig. 5C). The expression levels of the $\mathrm{H}_{2} \mathrm{~S}$-generating enzyme cystathionine $\gamma$-lyase (CSE) initially decrease, which reveals that the hepatic CSE is expressed to compensate for $\mathrm{H}_{2} \mathrm{~S}$ depletion (Fig. 5D). We also observed that the $\mathrm{H}_{2} \mathrm{~S}$ level drops significantly with the progression of liver injury, while ALT and AST levels are downregulated.This means that liver injury has gradually entered late stage and hepatocytes fail to selfrepair, eventually leading to cirrhosis. Histological changes from hematoxylin and eosin (H\&E) stain indicate liver rupture, cellular shrinkage, and neutrophil infiltration (Fig. S38†).

\section{Conclusions}

In summary, we developed the first example of a two-photon fluorescent COF nanoprobe, TpASH-NPHS, that shows favorable chemical stability and photostability, is conveniently synthesised, allows for versatile fluorescence modification, and exhibits a desirable nanoscale size. We have demonstrated the utility of TPASH-NPHS as a sensitive and selective fluorescent sensor of the gasotransmitter $\mathrm{H}_{2} \mathrm{~S}$. Our two-photon fluorescent COF serves to detect and image $\mathrm{H}_{2} \mathrm{~S}$ in live cells and deep tissues under NIR excitation, thereby greatly minimizing cellular autofluorescence, reducing tissue injury and increasing tissue penetration depth. Furthermore, the detection process is not jeopardized by haphazard interaction with endogenous enzymes, which holds great promise as an indicator for clinical applications; initial results with a cirrhotic mouse model confirm this. We are confident that our results encourage future research on employing fluorescent COF nanoprobes for biosensing and nanomedicine.

\section{Conflicts of interest}

There are no conflicts to declare.

\section{Acknowledgements}

This work was supported by the National Natural Science Foundation of China (Grants 21521063, 21325520, 21327009, and J1210040) and the Science and Technology Project of Hunan Province (2016RS2009 and 2016WK2002).

\section{Notes and references}

1 Z. Yang, A. Sharma, J. Qi, X. Peng, D. Y. Lee, R. Hu, D. Lin, J. Qu and J. S. Kim, Chem. Soc. Rev., 2016, 45, 4651-4667.

2 X. Wu, A. Shao, S. Zhu, Z. Guo and W. Zhu, Sci. China: Chem., 2016, 59, 62-69.

3 H. M. Kim and B. R. Cho, Chem. Rev., 2015, 115, 5014-5055. 4 X. Li, X. Gao, W. Shi and H. Ma, Chem. Rev., 2014, 114, 590659.

5 H. Zhu, J. Fan, J. Du and X. Peng, Acc. Chem. Res., 2016, 49, 2115-2126.
6 L. J. O'Connor, I. N. Mistry, S. L. Collins, L. K. Folkes, G. Brown, S. J. Conway and E. M. Hammond, ACS Cent. Sci., 2017, 3, 20-30.

7 Z. Wang, S. Chen, J. W. Lam, W. Qin, R. T. Kwok, N. Xie, Q. Hu and B. Z. Tang, J. Am. Chem. Soc., 2013, 135, 8238-8245.

8 J. Liang, G. Feng, R. T. K. Kwok, D. Ding, B. Tang and B. Liu, Sci. China: Chem., 2016, 59, 53-61.

9 C. S. Diercks and O. M. Yaghi, Science, 2017, 355, 1585.

10 N. Huang, P. Wang and D. Jiang, Nat. Rev. Mater., 2016, 1, 16068.

11 J. W. Colson, A. R. Woll, A. Mukherjee, M. P. Levendorf, E. L. Spitler, V. B. Shields, M. G. Spencer, J. Park and W. R. Dichtel, Science, 2011, 332, 228-231.

12 Y. Zhang, J. Duan, D. Ma, P. Li, S. Li, H. Li, J. Zhou, X. Ma, X. Feng and B. Wang, Angew. Chem., Int. Ed., 2017, 56, 16313-16317.

13 S. Karak, S. Kandambeth, B. P. Biswal, H. S. Sasmal, S. Kumar, P. Pachfule and R. Banerjee, J. Am. Chem. Soc., 2017, 139, 1856-1862.

14 G. Lin, H. Ding, R. Chen, Z. Peng, B. Wang and C. Wang, J. Am. Chem. Soc., 2017, 139, 8705-8709.

15 H. Ding, Y. Li, H. Hu, Y. Sun, J. Wang, C. Wang, C. Wang, G. Zhang, B. Wang, W. Xu and D. Zhang, Chem.-Eur. J., 2014, 20, 14614-14618.

16 Q. Sun, C. W. Fu, B. Aguila, J. Perman, S. Wang, H. Y. Huang, F. S. Xiao and S. Ma, J. Am. Chem. Soc., 2018, 140, 984-992.

17 S. Mitra, H. S. Sasmal, T. Kundu, S. Kandambeth, K. Illath, D. Diaz Diaz and R. Banerjee, J. Am. Chem. Soc., 2017, 139, 4513-4520.

18 G. Lin, H. Ding, D. Yuan, B. Wang and C. Wang, J. Am. Chem. Soc., 2016, 138, 3302-3305.

19 S. Y. Ding, M. Dong, Y. W. Wang, Y. T. Chen, H. Z. Wang, C. Y. Su and W. Wang, J. Am. Chem. Soc., 2016, 138, 30313037.

20 Y. Zhang, X. Shen, X. Feng, H. Xia, Y. Mu and X. Liu, Chem. Commun., 2016, 52, 11088-11091.

21 H. L. Qian, C. Dai, C. X. Yang and X. P. Yan, ACS Appl. Mater. Interfaces, 2017, 9, 24999-25005.

22 Y. Peng, Y. Huang, Y. Zhu, B. Chen, L. Wang, Z. Lai, Z. Zhang, M. Zhao, C. Tan, N. Yang, F. Shao, Y. Han and H. Zhang, J. Am. Chem. Soc., 2017, 139, 8698-8704.

23 I. Berlanga, M. L. Ruiz-Gonzalez, J. M. Gonzalez-Calbet, J. L. Fierro, R. Mas-Balleste and F. Zamora, Small, 2011, 7, 1207-1211.

24 D. N. Bunck and W. R. Dichtel, J. Am. Chem. Soc., 2013, 135, 14952-14955.

25 Z. Du, B. Song, W. Zhang, C. Duan, Y. L. Wang, C. Liu, R. Zhang and J. Yuan, Angew. Chem., Int. Ed., 2018, 57, 3999-4004.

26 G. Xu, Q. Yan, X. Lv, Y. Zhu, K. Xin, B. Shi, R. Wang, J. Chen, W. Gao, P. Shi, C. Fan, C. Zhao and H. Tian, Angew. Chem., Int. Ed., 2018, 57, 3626-3630.

27 Y. L. Pak, J. Li, K. C. Ko, G. Kim, J. Y. Lee and J. Yoon, Anal. Chem., 2016, 88, 5476-5481.

28 J. Kang, Z. Li, C. L. Organ, C. M. Park, C. T. Yang, A. Pacheco, D. Wang, D. J. Lefer and M. Xian, J. Am. Chem. Soc., 2016, 138, 6336-6339. 
29 X. Jiao, Y. Xiao, Y. Li, M. Liang, X. Xie, X. Wang and B. Tang, Anal. Chem., 2018, 90, 7510-7516.

30 Y. Ma, C. Zhang, P. Yang, X. Li, L. Tong, F. Huang, J. Yue and B. Tang, Nanoscale, 2018, 10, 15793-15798.

31 G. J. Mao, T. T. Wei, X. X. Wang, S. Y. Huan, D. Q. Lu, J. Zhang, X. B. Zhang, W. Tan, G. L. Shen and R. Q. Yu, Anal. Chem., 2013, 85, 7875-7881.

32 A. R. Lippert, E. J. New and C. J. Chang, J. Am. Chem. Soc., 2011, 133, 10078-10080.

33 M. D. Hammers, M. J. Taormina, M. M. Cerda, L. A. Montoya, D. T. Seidenkranz, R. Parthasarathy and M. D. Pluth, J. Am. Chem. Soc., 2015, 137, 10216-10223.

34 L. Zhou, X. Zhang, Q. Wang, Y. Lv, G. Mao, A. Luo, Y. Wu, Y. Wu, J. Zhang and W. Tan, J. Am. Chem. Soc., 2014, 136, 9838-9841.

35 H. W. Liu, X. B. Zhang, J. Zhang, Q. Q. Wang, X. X. Hu, P. Wang and W. Tan, Anal. Chem., 2015, 87, 8896-8903.

36 P. Wang, C. Zhang, H. W. Liu, M. Xiong, S. Y. Yin, Y. Yang, X. X. Hu, X. Yin, X. B. Zhang and W. Tan, Chem. Sci., 2017, 8, 8214-8220.

37 S. Xu, H. W. Liu, X. X. Hu, S. Y. Huan, J. Zhang, Y. C. Liu, L. Yuan, F. L. Qu, X. B. Zhang and W. Tan, Anal. Chem., 2017, 89, 7641-7648.
38 H. W. Liu, Y. Liu, P. Wang and X. B. Zhang, Methods Appl. Fluoresc., 2017, 5, 012003.

39 J. Li, C. S. Lim, G. Kim, H. M. Kim and J. Yoon, Anal. Chem., 2017, 89, 8496-8500.

40 Z. R. Dai, G. B. Ge, L. Feng, J. Ning, L. H. Hu, Q. Jin, D. D. Wang, X. Lv, T. Y. Dou, J. N. Cui and L. Yang, J. Am. Chem. Soc., 2015, 137, 14488-14495.

41 B. Zhang, X. Yang, R. Zhang, Y. Liu, X. Ren, M. Xian, Y. Ye and Y. Zhao, Anal. Chem., 2017, 89, 10384-10390.

42 M. A. Khayum, S. Kandambeth, S. Mitra, S. B. Nair, A. Das, S. S. Nagane, R. Mukherjee and R. Banerjee, Angew. Chem., Int. Ed., 2016, 55, 15604-15608.

43 D. A. Vazquez-Molina, G. S. Mohammad-Pour, C. Lee, M. W. Logan, X. Duan, J. K. Harper and F. J. Uribe-Romo, J. Am. Chem. Soc., 2016, 138, 9767-9770.

44 J. L. Wallace and R. Wang, Nat. Rev. Drug Discovery, 2015, 14, 329-345.

45 T. B. Ren, W. Xu, Q. L. Zhang, X. X. Zhang, S. Y. Wen, H. B. Yi, L. Yuan and X. B. Zhang, Angew. Chem., Int. Ed., 2018, 57, 7473-7477.

46 G. Tan, S. Pan, J. Li, X. Dong, K. Kang, M. Zhao, X. Jiang, J. R. Kanwar, H. Qiao, H. Jiang and X. Sun, PLoS One, 2011, 6, 25943. 